\title{
Nanofibril Self-assembly of an Arylene Ethynylene Macrocycle
}

Kaushik Balakrishnan, ${ }^{1}$ Aniket Datar, ${ }^{1}$ Wei Zhang, ${ }^{2}$ Xiaomei Yang, ${ }^{1}$ Tammene Naddo, ${ }^{1}$ Jialing Huang, ${ }^{3}$ Jianmin Zuo, ${ }^{4}$ Max Yen, ${ }^{3}$ Jeffrey S. Moore, ${ }^{*}, 2$ and Ling Zang*,1

${ }^{1}$ Department of Chemistry and Biochemistry, Southern Illinois University, Carbondale, IL 62901;

${ }^{2}$ Departments of Chemistry and Materials Science and Engineering, 600 South Mathews Avenue, University of Illinois at Urbana-Champaign, Urbana, Illinois 61801; ${ }^{3}$ Materials Technology Center (MTC), Southern Illinois University, Carbondale, IL 62901; ${ }^{4}$ Department of Materials Science and Engineering, University of Illinois at Urbana-Champaign, Urbana, Illinois 61801.

\section{Supporting Information}

\section{Materials and general methods}

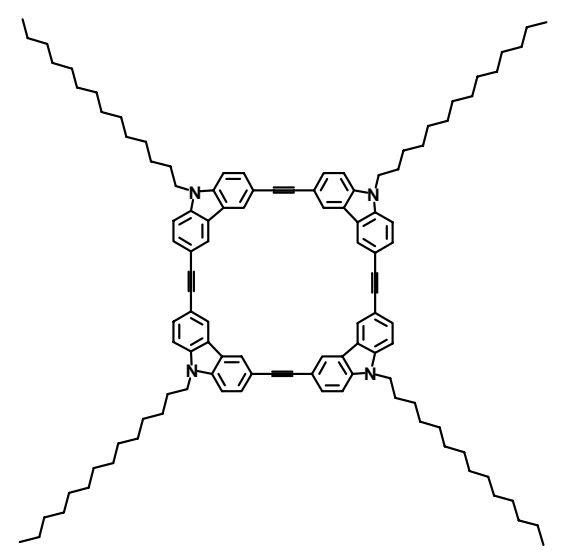

The AEM molecule, 1.

Synthesis and characterization of $\mathbf{1}$ were followed the method previously developed in the Moore lab. ${ }^{1}$ The starting materials and all solvents (HPLC or spectroscopic grade) were purchased from Fisher and Aldrich, and used as received.

UV-vis absorption and fluorescence spectra were measured on a PerkinElmer Lambda 25 spectrophotometer and LS 55 fluorometer, respectively. The fluorescence spectra of the solid state samples were recorded with a modular fluorometer (Photon Technology International Inc.) which allows for highly sensitive measurement of the low emission of solid state.

Pyrex glass cover slips (from Corning Inc.) were used as the support substrate for AFM measurement of the self-assembled nanostructures. Prior to use, a cover slip was cleaned with piranha reagent $\left(30: 70 \mathrm{H}_{2} \mathrm{O}_{2}(35 \%): \mathrm{H}_{2} \mathrm{SO}_{4}\right)$, followed by rinsing with water and methanol. ${ }^{2}$ Caution: Piranha solution is an extremely strong oxidizing reagent. The roughness of the glass surface thus cleaned was about $0.8 \mathrm{~nm}$ (Figure S1) as confirmed by the shear-force mode topography measurement performed with a scanning probe microscope (Aurora III, Veeco). Such a flat surface is suitable for AFM measurement of the nanofibrils with thickness of only a few nanometers. 


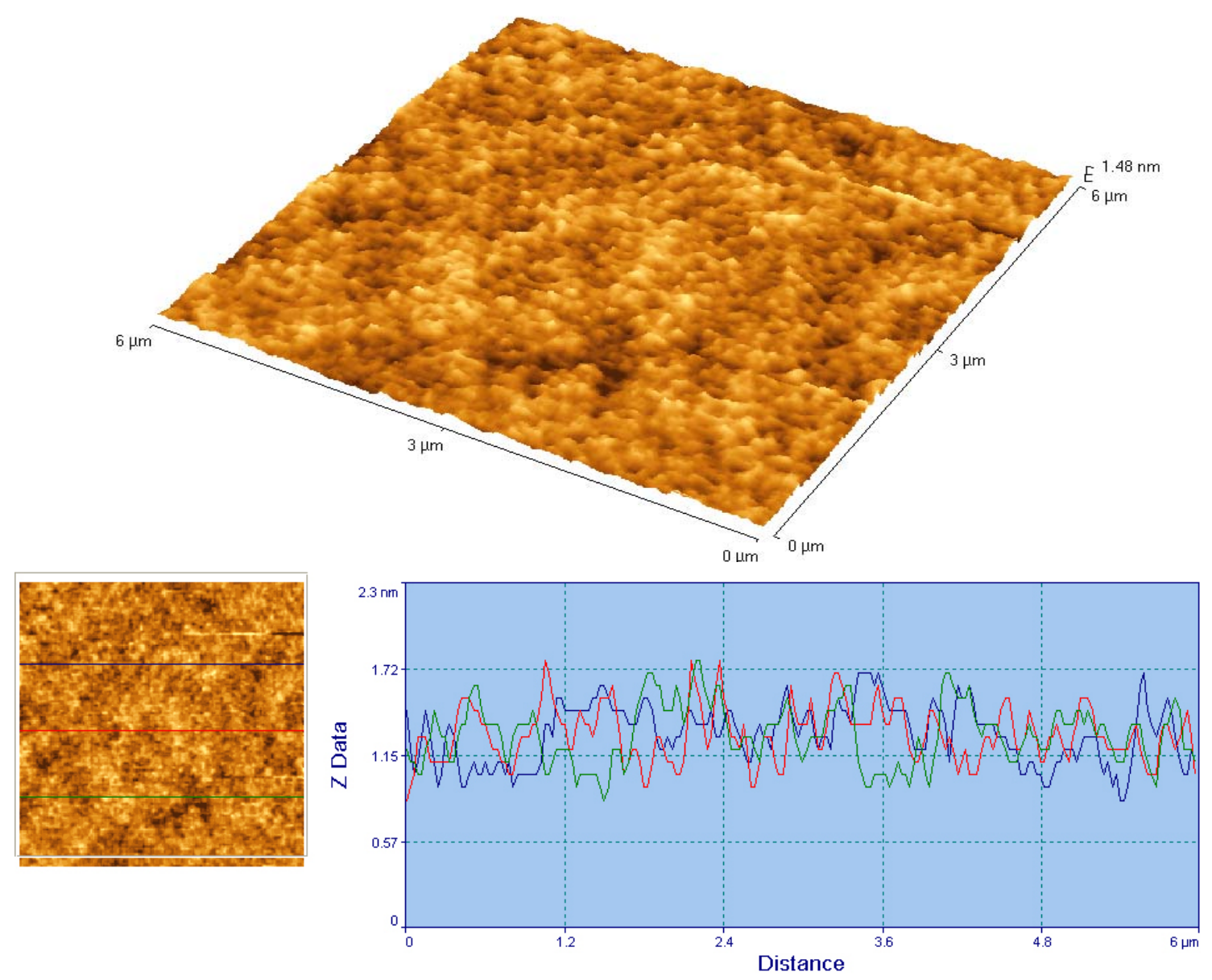

Figure S1. (top) 3D AFM image of a glass surface cleaned by piranha reagent; (bottom) the same image with three line-scan profiles showing the roughness $(\sim 0.8 \mathrm{~nm})$ of the surface.

\section{Nanofibril fabrication via gelation}

Self-assembly of the nanofibrils of $\mathbf{1}$ was performed through a gelation process in cyclohexane. Briefly, a certain amount of $\mathbf{1}(4 \mathrm{mg} / \mathrm{mL})$ was added to cyclohexane, followed by sonication for $5 \mathrm{~min}$. A milk-like suspension was thus obtained (Figure S2A). Upon heating in an oil bath at $100{ }^{\circ} \mathrm{C}$, the suspension became totally dissolved in a few min, producing a transparent solution in light brown color (Figure S2B). With cooling in air (room temperature), the solution turned to be little turbid within 2 min as the molecular aggregation started (Figure S2C). After about $5 \mathrm{~min}$, the gelation started as revealed by the phase immobilization (Figure S2D). Leaving the sample open in a hood led to the formation of an aged gel within about one hour (Figure S2E).

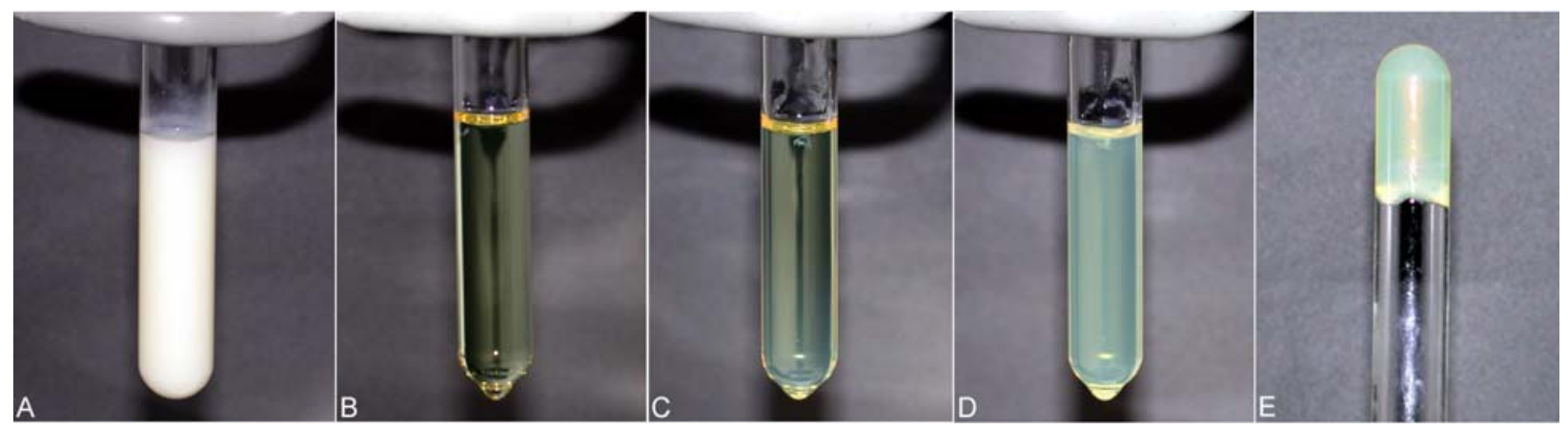

Figure S2. Samples of $\mathbf{1}$ at different stages of the gelation process. (A) a milky suspension of 1 in cyclohexane $(4 \mathrm{mg} / \mathrm{mL})$ after sonication; (B) totally dissolved after heating at $100{ }^{\circ} \mathrm{C}$ in an oil bath; (C) 
about 2 min after cooling in air; (D) about 5 min after cooling in air; (E) the gel formed after cooling and aging in air for $1 \mathrm{~h}$.

The gel thus made is very tender, and can be transferred simply by pipetting. The sample for AFM measurement was prepared by spin-casting one drop (of disposal glass pipette) of the gel onto a cleaned glass cover slip at $2500 \mathrm{rpm}$. For TEM measurement (which demands minimal deposition of sample to afford sufficient electron transmission), the gel had to be diluted with a proper solvent before being deposited onto the copper grids. See section IV for little more details of the preparation of TEM samples.

\section{AFM characterization of nanofibrils}

AFM measurement was carried out in tapping mode on a TopoMetrix Explorer using antimony doped silicon tip. The largest scanning area is $50 \times 50 \mu \mathrm{m}$, and the highest z-resolution is about $0.2 \mathrm{~nm}^{3}$ In addition to the images shown in Figure 1, Figure S3 shows more large-area scanning images of the nanofibrils formed by direct casting of the gel onto glass. The line-scan profiles shown in Figure S4 clearly reveal the thickness of the nanofibrils and the bundles. In Figure S4C, two entangled small fibrils of only $6.3 \mathrm{~nm}$ thickness were clearly imaged. The thickness corresponds to a cross-section composed of three molecules of $\mathbf{1}$ (see section V, Figure S8).

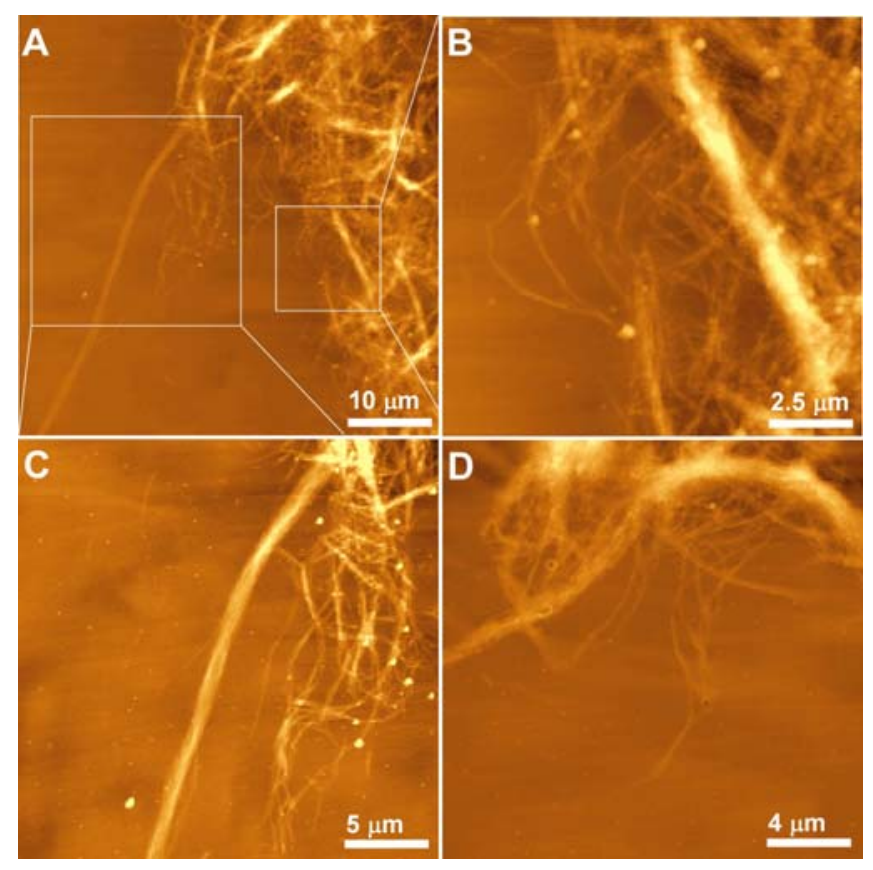

Figure S3. Large-area AFM images of the gel of 1 spin-cast and dried on glass. (B) and (C) show the two zoomed-in areas marked in A. The total z-height ranges for A-D are 250, 150, 58 and $130 \mathrm{~nm}$, respectively. 

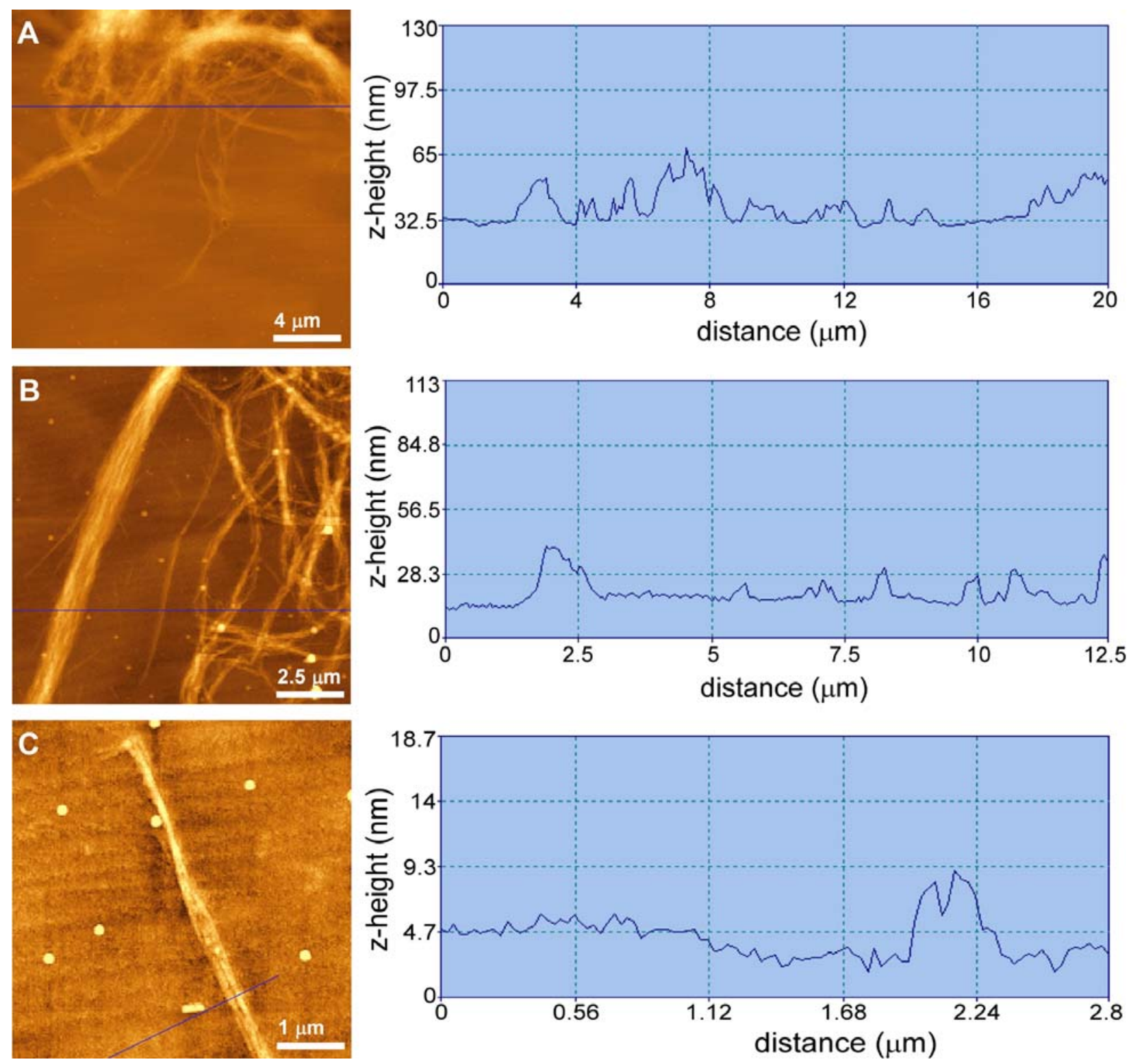

Figure S4. Line-scan profiles for two large-area AFM images (A and B) and a zoomed-in image over two entangled nanofibers $(\mathrm{C})$.

\section{TEM characterization of nanofibrils}

TEM measurement was performed with a Hitachi 7100 (operated at $100 \mathrm{kV}$ ). The sample was prepared by drop-casting a diluted gel suspension onto a holey carbon or silicon oxide film, followed by drying in air. The dilution of gel was carried out in both hexane and cyclohexane. As depicted in Figure 2 , dilution in cyclohexane provided quite well dispersion of nanofibrils from the gel. Dilution in hexane produced similar fibril dispersion, except for formation of some particulate aggregates (Figure S5). The formation of particles is likely due to the lower solubility of $\mathbf{1}$ in hexane.

In contrast, dilution of the gel into a 'poor' solvent (e.g., methanol) produced only chunky aggregates (Figure S6), rather than well-dispersed fibrils. This is consistent with the observation of direct dispersion of a chloroform solution of $\mathbf{1}$ into methanol, in which particulate aggregates were formed (see section VI). The formation of chunky aggregate in methanol is likely the result of the limited solubility of $\mathbf{1}$ in the solvent. 

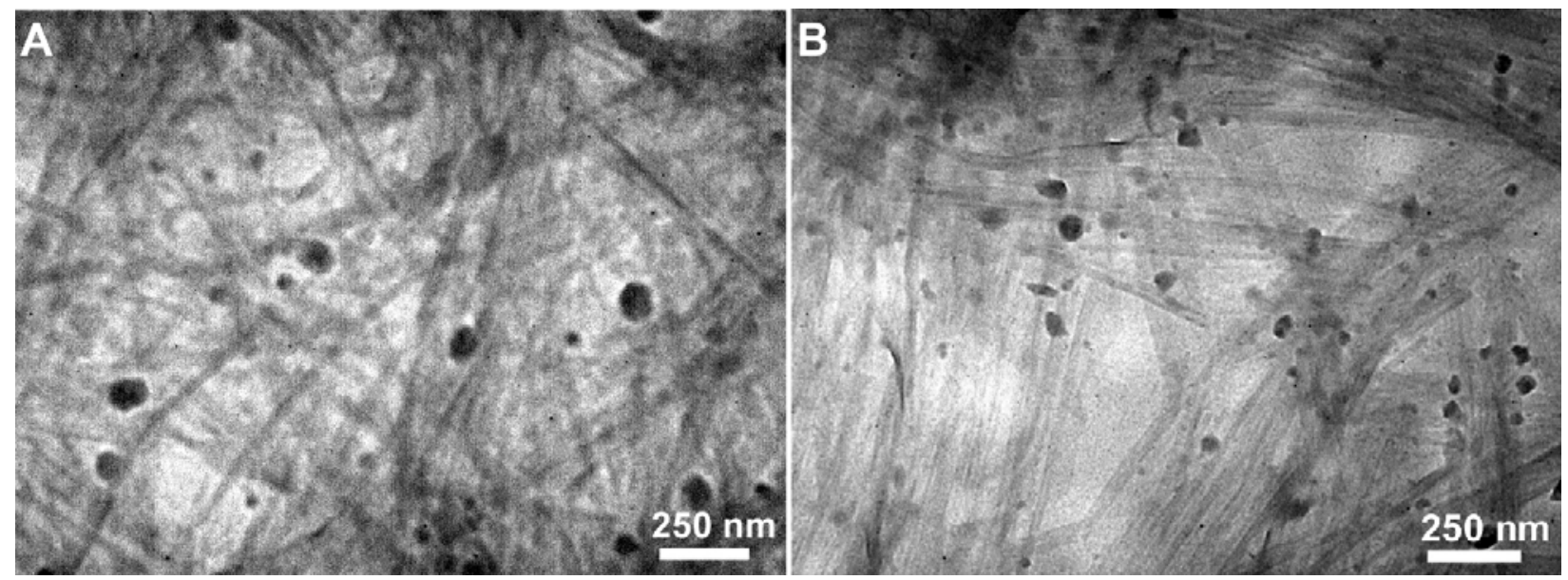

Figure S5. TEM images of the gel of $\mathbf{1}$ deposited on silicon oxide (A) and holey carbon (B) films. The TEM samples were prepared by drop-casting of a diluted gel suspension in hexane ( $\sim 5 \%$ vol dilution).

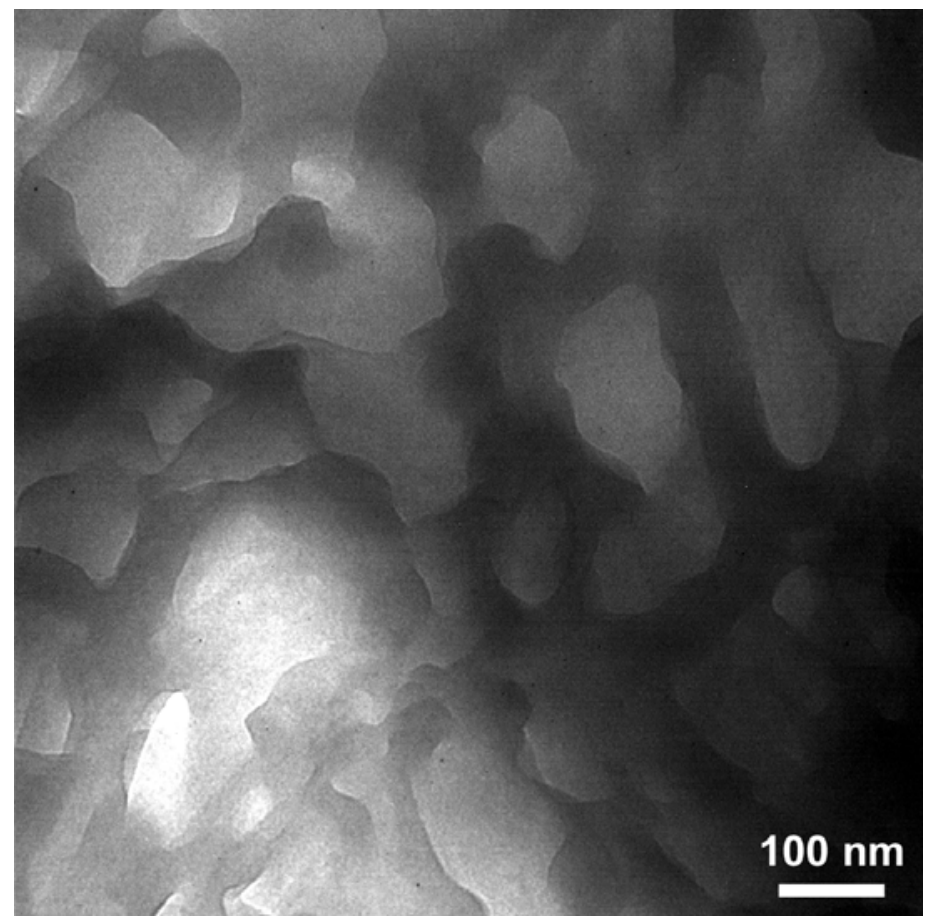

Figure S6. TEM image of the chunky aggregates of 1 formed by dispersion of the gel into methanol $(\sim$ $5 \%$ vol dilution). The sample was deposited and dried on silicon oxide film.

\section{X-ray diffraction of nanofibrils}

X-ray diffraction was performed on a Rigaku D/Max-b, for which a $\mathrm{Cu}-\mathrm{K} \alpha$ radiation $(\lambda=1.54 \AA)$ was used. The sample was prepared by drop casting the gel on a glass cover slip, followed by drying at room temperature. Figure S7 shows the wide-angle diffraction pattern of the dried gel deposited on glass. The diffractogram is dominated by a single sharp peak at low angle $\left(2 \theta 4.1^{\circ}\right)$, indicative of columnar assemblies. ${ }^{4}$ A d-spacing of $21.6 \AA$ is calculated from the angle of this primary diffraction peak. Based on this measurement and the dimensional size of the molecule (obtained from DFT configuration optimization with Gaussian 03, B3LYP/6-31g*), a 2D supramolecular assembly of 1 is schematically depicted in Figure S8. Such a supra-lattice structure has been observed (by STM) for other AEM molecules (in hexagonal shape) deposited as monolayer on flat substrates. ${ }^{5}$

The nanofibril structure obtained in this study is likely formed by the 1D growth (stacking) of the supra-lattice network via $\pi-\pi$ interaction between the large $\pi$-systems. The $d$-spacing corresponding to the $\pi$-stacking is inferred from the peak at $2 \theta 23.2^{\circ}$, and calculated to be ca. $3.8 \AA$, which is quite close 
to the typical distance $(\sim 3.5 \AA)$ for an effective $\pi-\pi$ stacking between aromatic molecules. ${ }^{4,6-9}$ Considering the strong hydrophobic association between the long alkyl side-chains, the 2D supra-lattice could be formed by just a few molecules, thus producing a fiber of cross-section in the size range of a few nanometers. Indeed, such small fibrils were formed in the gelation of $\mathbf{1}$, as revealed by AFM imaging (Figure 1C and D, Figure S4C). The stability of such small molecular assemblies is dominated by the cooperation between the side-chain association and the strong inter-planar $\pi-\pi$ interaction.

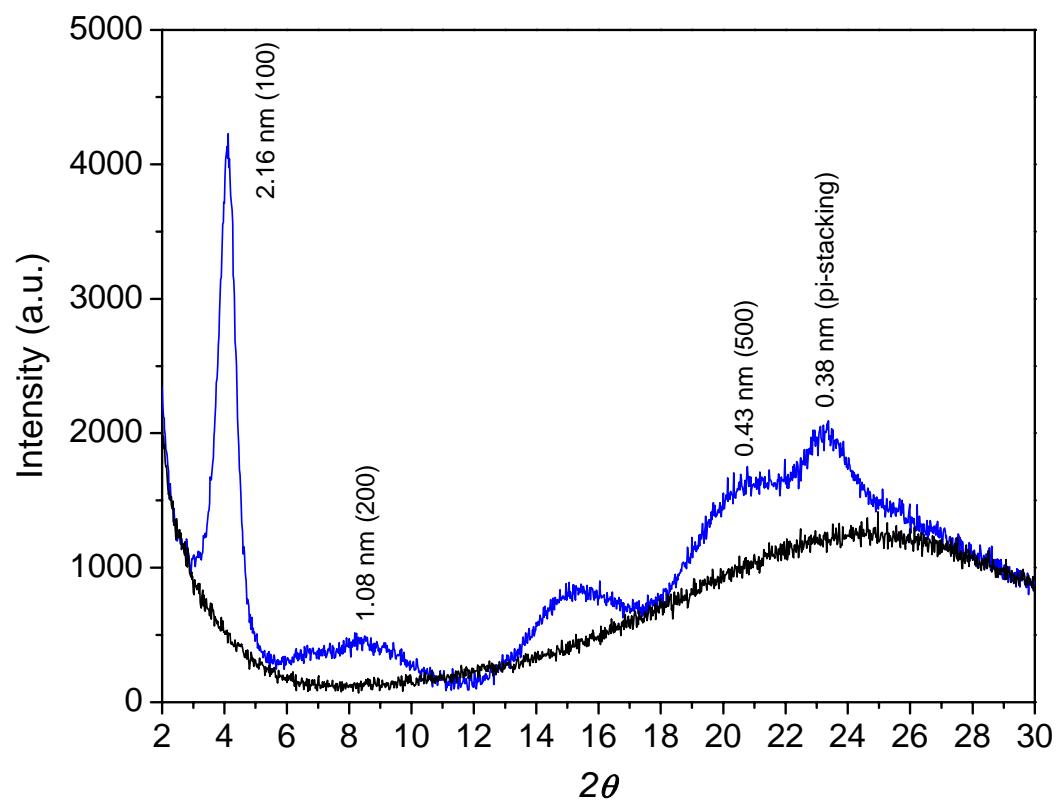

Figure S7. X-ray diffraction pattern (blue line) of the gel of 1 deposited on glass. Also shown for comparison is the diffraction pattern of the sample made by direct deposition of a THF solution of 1 on glass, which depicts no diffraction peaks, consistent with the amorphous phase formed by the fast evaporation on surface. The broad background diffraction is due to the glass substrate.

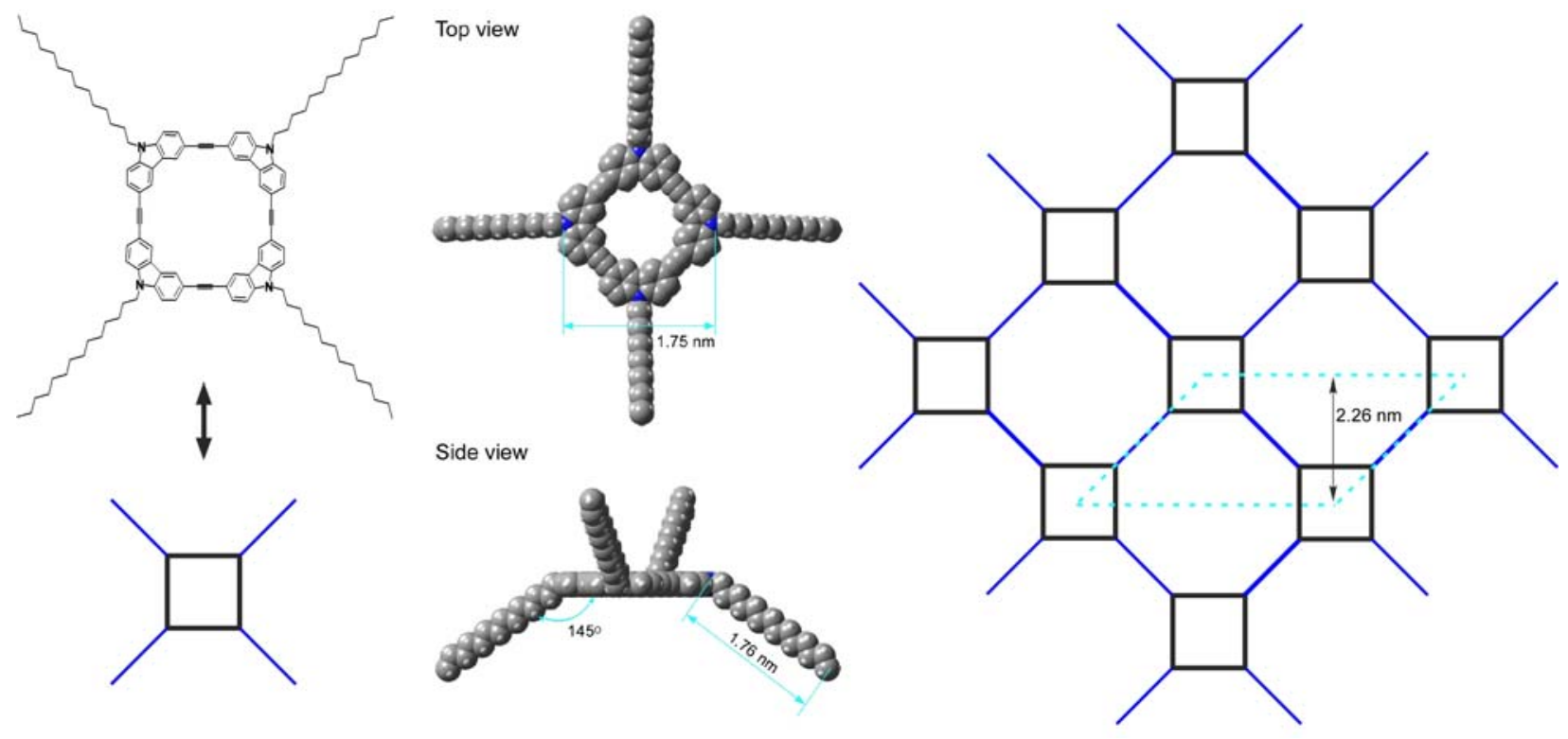

Figure S8. Energy minimized configuration of 1 obtained by DFT calculation (B3LYP/6-31g*) using Gaussian 03, and the proposed one-layer supra-molecular assembly through side-chain association. The $\mathrm{d}$-spacing for an assembly with full side-chain interdigitation is calculated as $2.26 \mathrm{~nm}$, which is quite 
consistent with the value, $2.16 \mathrm{~nm}$, measured by the X-ray diffraction (Figure S7). The slight difference might be due to the chain folding and the $\pi-\pi$ stacking offset in the real assembly.

\section{Self-assembly of 1 in solutions: a comparative investigation}

As a comparison, the self-assembly of $\mathbf{1}$ was also performed in solution using a so-called 'phase transfer' method, which has been developed in our lab for assembling large, planar aromatic molecules into 1D nanostructures (e.g., nanowires). ${ }^{10}$ Briefly, the molecular assembly was processed through slow crystallization at the interface between a 'good' and a 'poor' solvent, where the slow 'phase transfer' between the two solvents decreases the solubility at the interface. The poor solvent (e.g., methanol) is normally quite different (e.g., in term of polarity) from the good solvent (e.g., chloroform), thus providing the possibility to keep the two solvents in separate phases for an extended period. Typically, a larger amount (10:1 vol) of 'poor' solvent was transferred atop a concentrated chloroform solution of the molecule $(1 \mathrm{mM})$ in a test tube. Within minutes, crystallization (precipitation) occurred at the interface, followed by slow diffusion into the upper phase of the 'poor' solvent. The crystals thus formed can be transferred and cast onto glass surface by pipetting.

In this study, the 'good' solvent used was chloroform, and two other solvents (methanol and acetonitrile) were used as the 'poor' solvent. For both the two solvents, the self-assembly produced particles (Figure S9 and 10), rather than 1D structures. In general, the particles formed in acetonitrile were larger than those formed in methanol, likely due to the lower solubility (and thus faster aggregation) of $\mathbf{1}$ in acetonitrile.

These observations implied that formation of 1D molecular assembly demands a fabrication condition in which the inter-planar $\pi-\pi$ stacking is highly favored over the lateral side-chain association. Gelation has proven to be an effective approach to 1D self-assembly, mainly because the gradual cooling process favors the $\pi-\pi$ stacking, and meanwhile the decreased mobility of molecules minimizes the steric hindrance of side-chains.
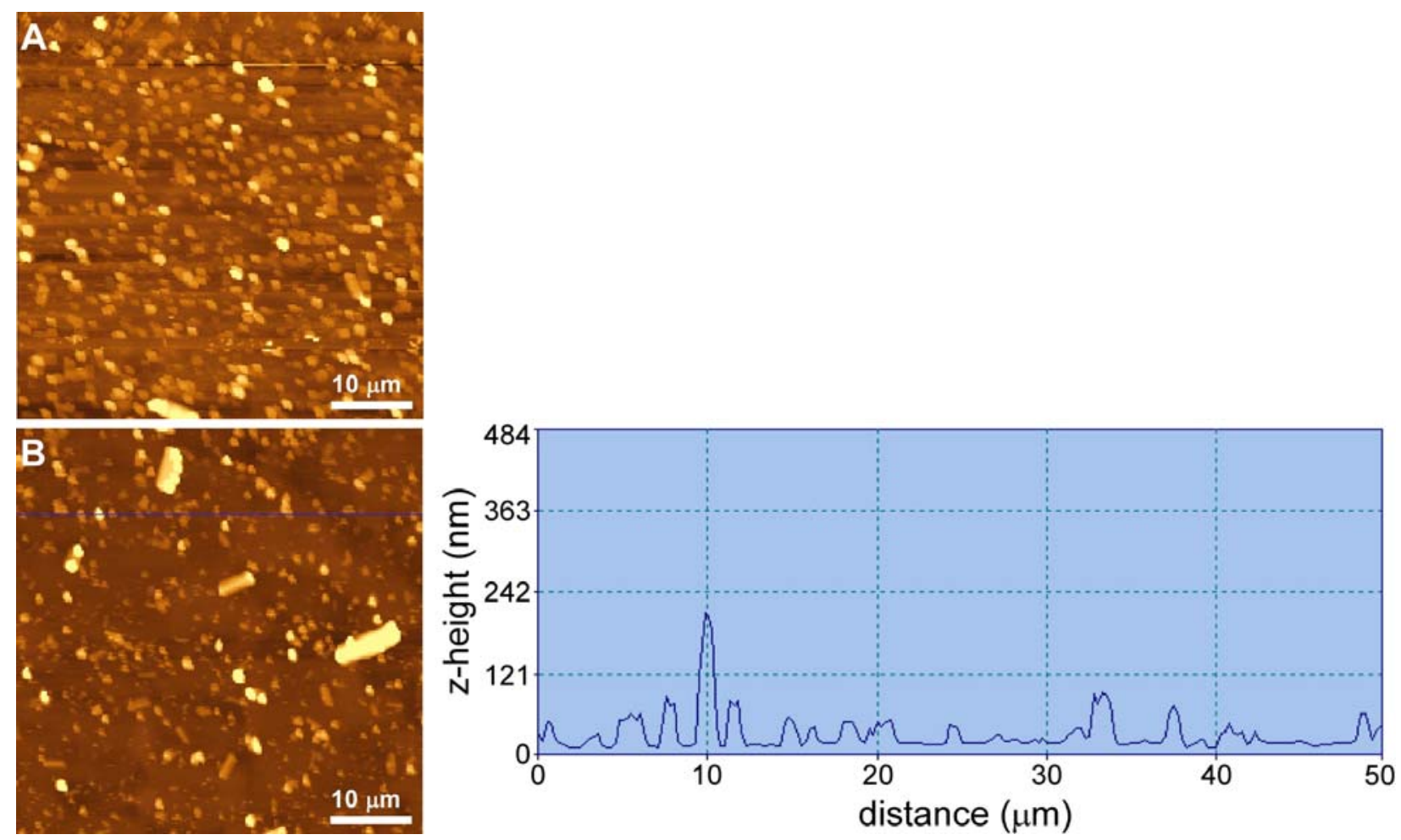

Figure S9. Large-area AFM images of nanocrystals of 1 prepared by the phase-transfer crystallization between excessive methanol (10:1 vol) and a concentrated chloroform solution $(1 \mathrm{mM})$. The sample was 
prepared by drop-casting the methanol suspension onto a glass cover slip. Most of the nanocrystals obtained are in nearly spherical shape, while some nanorods are occasionally observed.
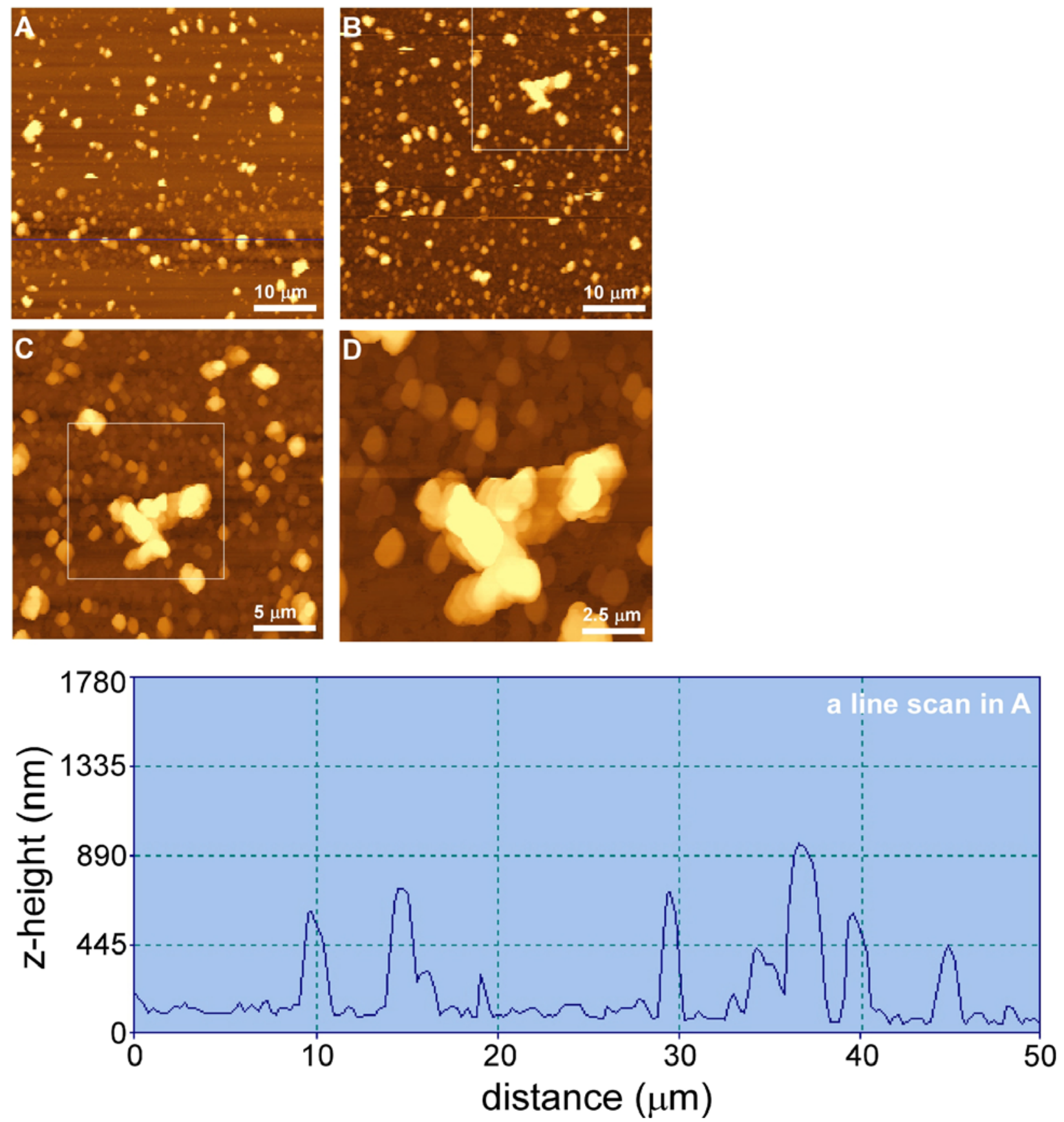

Figure S10. (A, B) Large-area AFM images of nanocrystals of 1 prepared by the phase-transfer crystallization between excessive acetonitrile (10:1 vol) and a concentrated chloroform solution (1 mM); (C, D) zoomed-in images of the area marked in B. The sample was prepared by drop-casting the acetonitrile suspension onto a glass cover slip. Compared to the assembly prepared from methanol (Figure S9), the nanocrystals obtained in acetonitrile are wholly in particulate morphology, with no formation of nanorods.

(1) Zhang, W.; Moore, J. S. J .Am. Chem. Soc 2004, 126, 12796.

(2) Zang, L.; Liu, R.; Holman, M. W.; Nguyen, K. T.; Adams, D. M. J. Am. Chem. Soc. 2002, 124, 10640-10641.

(3) Datar, A.; Oitker, R.; Zang, L. Chem. Commun. 2006, in press.

(4) Bushey, M. L.; Hwang, A.; Stephens, P. W.; Nuckolls, C. J. Am. Chem. Soc. 2001, 123, 8157-8158. 
(5) Pan, G.-B.; Cheng, X.-H.; Hoeger, S.; Freyland, W. J. Am. Chem. Soc., ACS ASAP.

(6) Ajayaghosh, A.; George, S. J. J. Am. Chem. Soc. 2001, 123, 5148-5149.

(7) Endo, K.; Ezubara, T.; Koyanagi, M.; Masuda, H.; Aoyama, Y. J. Am. Chem. Soc. 1997, 119, 499-505.

(8) Weck, M.; Dunn, A. R.; Matsumoto, K.; Coates, G. W.; Lobkovsky, E. B.; Grubbs, R. H. Angew. Chem. Int. Ed. 1999, 38, 2741-2745.

(9) Struijk, C. W.; Sieval, A. B.; Dakhorst, J. E. J.; van Dijk, M.; Kimkes, P.; Koehorst, R. B. M.; Donker, H.; Schaafsma, T. J.; Picken, S. J.; van de Craats, A. M.; Warman, J. M.; Zuihof, H.; Sudholter, E. J. R. J. Am. Chem. Soc. 2000, 122, 11057-11066.

(10) Datar, A.; Balakrishnan, K.; Yang, X. M.; Zuo, X.; Huang, J. L.; Yen, M.; Tiede, D. M.; Zang, L. Angew. Chem. Int. Ed. 2006, submitted. 